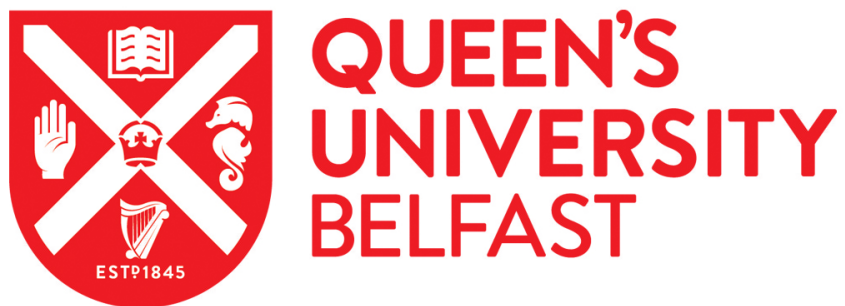

\section{Experimental investigation of a wavelet based drive-by bridge inspection system incorporating pattern recognition}

McGetrick, P. J., \& Kim, C. W. (2014). Experimental investigation of a wavelet based drive-by bridge inspection system incorporating pattern recognition. 333-340. Paper presented at 4th International Symposium on LifeCycle Civil Engineering (IALCCE 2014), Tokyo, Japan.

http://www.f.waseda.jp/akiyama617/ialcce2014/contents/mini_symposia.html

Document Version:

Peer reviewed version

Queen's University Belfast - Research Portal:

Link to publication record in Queen's University Belfast Research Portal

Publisher rights

Copyright 2014 International Association for Life-Cycle Civil Engineering.

\section{General rights}

Copyright for the publications made accessible via the Queen's University Belfast Research Portal is retained by the author(s) and / or other copyright owners and it is a condition of accessing these publications that users recognise and abide by the legal requirements associated with these rights.

Take down policy

The Research Portal is Queen's institutional repository that provides access to Queen's research output. Every effort has been made to ensure that content in the Research Portal does not infringe any person's rights, or applicable UK laws. If you discover content in the Research Portal that you believe breaches copyright or violates any law, please contact openaccess@qub.ac.uk. 


\title{
Experimental investigation of a wavelet based drive-by bridge inspection system incorporating pattern recognition
}

\author{
P.J. McGetrick \\ SPACE, David Keir Building, Queen's University Belfast, BT9 5AG, UK \\ C.W. Kim \\ Dept. of Civil and Earth Resources Engineering, Kyoto University, Kyoto 615-8540, Japan
}

\begin{abstract}
This paper presents the results of an experimental investigation, carried out in order to verify the feasibility of a 'drive-by' approach which uses a vehicle instrumented with accelerometers to detect and locate damage in a bridge. In theoretical simulations, a simplified vehicle-bridge interaction model is used to investigate the effectiveness of the approach in detecting damage in a bridge from vehicle accelerations. For this purpose, the accelerations are processed using a continuous wavelet transform and damage indicators are evaluated and compared. Alternative statistical pattern recognition techniques are incorporated to allow for repeated vehicle passes. Parameters such as vehicle speed, damage level, location and road roughness are varied in simulations to investigate the effect. A scaled laboratory experiment is carried out to assess the effectiveness of the approach in a more realistic environment, considering a number of bridge damage scenarios.
\end{abstract}

\section{INTRODUCTION}

In recent years, with the on-going development of effective techniques for the monitoring of the condition of infrastructure such as bridges, important considerations include the operational cost and practical implementation of such techniques. Over a bridge's lifetime, it will suffer from deterioration and existing techniques (Carden \& Fanning 2004, Boller et al. 2009) can be effective in providing early warning if a bridge's condition becomes unsafe. However, they can also be costly, labour intensive and hence time consuming, in addition to involving practical restrictions related to the fitting of sensors and acquisition electronics directly onto the bridge. This paper presents an indirect wavelet-based inspection system which aims to provide an efficient, low cost alternative for the monitoring of bridges, consisting of a vehicle fitted with accelerometers on its axles. All of the sensors and electronics are contained within the vehicle hence it targets an easy to implement 'driveby' approach which can be employed at highway speeds to address the large proportion of existing short to medium span highway bridges that are not instrumented. Effective implementation of this approach would reduce the need for visual inspections and direct instrumentation of bridge while also reducing maintenance costs.

This type of indirect approach, focusing on the dynamic response of a vehicle passing over the bridge, has been investigated by a number of researchers in recent years. In theoretical (Yang et al.
2004, Bu et al. 2006, McGetrick et al. 2009, Yang \& Chang 2009, González et al. 2012, Keenahan et al. 2012), experimental (Lin \& Yang 2005, Kim et al. 2011) and field investigations (González et al. 2008, Miyamoto \& Yabe 2012), the feasibility of the identification of bridge properties from the dynamic response of the vehicle has been verified, highlighting that sufficient dynamic excitation of the bridge and the road pavement roughness in particular are important factors affecting the effectiveness of the indirect approach.

The drive-by system investigated in this paper is based on the use of wavelet theory (Reda Taha et al. 2006), in particular focusing on the continuous wavelet transform (CWT). Numerical investigations of indirect approaches incorporating wavelet theory have been carried out in order to identify localized damage within a bridge; Nguyen \& Tran (2010) and Khorram et al. (2012) used the wavelet transform of vehicle displacements, highlighting that such an approach can be more effective than using sensors directly on the bridge. In a parametric study, McGetrick \& Kim (2013) focused on the CWT of vehicle accelerations; noting in theoretical simulations that low speeds provide higher accuracy for damage detection, while in an experimental investigation it was difficult to accurately identify the damage location. McGetrick \& Kim (2014) investigated the use of a pattern-adapted wavelet basis for the CWT of vehicle accelerations, highlighting the potential of the CWT to be incorporated in statistical pattern recognition (SPR) methods for structural 
damage detection (Mesa 2005, Nair \& Kiremedjian 2009). It is found that compared to a Morlet CWT, the pattern-adapted CWT was more sensitive to the damage location in a bridge but slightly less sensitive to the damage level.

This paper further extends the study by McGetrick \& Kim (2013) to different damage indicators (DI), introduces the use of an alternative statistical pattern recognition technique and compares damage detection results for the drive-by system with those of the original approach study. For this purpose, vehicle-bridge interaction (VBI) models are used in theoretical simulations and in a laboratory experiment.

\section{METHODOLOGY}

\subsection{Theoretical simulation model}

The VBI model described by McGetrick et al. (2013) is used in theoretical simulations (Figure 1). An 18 tonne two-axle vehicle is represented by a 4 degree of freedom half-car model and it crosses at constant speed over a finite element (FE) beam representing the bridge. The vibration of the vehicle and beam are coupled at their points of contact; at $x_{1}$ and $x_{2}$ in Figure 1 . The body and axle masses of the vehicle are $m_{s}=16200 \mathrm{~kg}, m_{u, 1}=700 \mathrm{~kg}$ and $m_{u, 2}=1100 \mathrm{~kg}$ respectively. The air suspension and tire spring linear stiffness coefficients for axle 1 are $K_{s, 1}=4 \times 10^{5}$ $\mathrm{N} / \mathrm{m}$ and $\mathrm{K}_{t, 1}=1.75 \times 10^{6} \mathrm{~N} / \mathrm{m}$ respectively while the corresponding values for axle 2 are $K_{s, 2}=1 \times 10^{6}$ $\mathrm{N} / \mathrm{m}$ and $K_{t, 2}=3.5 \times 10^{6} \mathrm{~N} / \mathrm{m}$ respectively. The suspension viscous damping coefficients are $C_{s, 1}=10 \times$ $10^{3} \mathrm{Ns} / \mathrm{m}$ and $C_{s, 2}=20 \times 10^{3} \mathrm{Ns} / \mathrm{m}$. The sprung mass moment of inertia, $I_{s}$, is $93234 \mathrm{kgm}^{2}$. The distances of the axles to the centre of gravity, $o$, are $D_{1}=2.85$ $\mathrm{m}$ and $D_{2}=1.9 \mathrm{~m}$. Finally, the vehicle's frequencies of vibration are $f_{v, 1}=1.1 \mathrm{~Hz}, f_{v, 2}=1.4 \mathrm{~Hz}, f_{v, 3}=8.8$ $\mathrm{Hz}$ and $f_{v, 4}=10.2 \mathrm{~Hz}$ corresponding to body bounce, body pitch and the hop of axles 1 and 2 respectively.

A bridge of span length $15 \mathrm{~m}$ is used in simulations. The healthy bridge has elemental stiffness, $E J_{0}$, of $1.846 \times 10^{10} \mathrm{~N} \mathrm{~m}^{2}$, mass per unit length, $\mu$, of $28125 \mathrm{~kg} / \mathrm{m}$, damping of $3 \%$ and a first natural frequency, $f_{b, 1}$, of $5.66 \mathrm{~Hz}$. To damage the beam, percentage stiffness reductions are applied to individual elements, representing localised damage within the bridge. In this paper, damage is applied to elements at $L / 2$ and $3 L / 8$ separately.

The coupled VBI system is solved using the Wilson-theta integration scheme (Tedesco et al. 1999) and a sampling frequency of $100 \mathrm{~Hz}$ is used in simulations. Acceleration measurements, $\ddot{y}_{s, i}$, are recorded above the axles as shown in Figure 1.

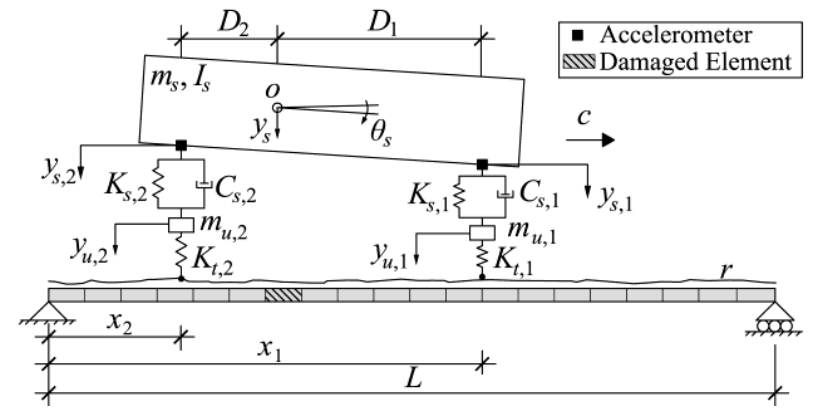

Figure 1. Theoretical vehicle-bridge interaction model.

\subsection{Experimental model}

A scaled two-axle vehicle model and steel beam were adopted as the VBI model in the laboratory experiment and this experimental setup is illustrated in Figure 2.

The vehicle had an axle spacing of $0.4 \mathrm{~m}$ and was fitted with accelerometers at the centre of each axle (Figure 2(c)). A wireless router and data logger allowed accelerations to be recorded remotely. A constant vehicle speed was maintained by an electronic controller and each bridge crossing was repeated 5 times. Three speeds were adopted; $\mathrm{S} 1=0.93 \mathrm{~m} / \mathrm{s}, \mathrm{S} 2$ $=1.16 \mathrm{~m} / \mathrm{s}$ and $\mathrm{S} 3=1.63 \mathrm{~m} / \mathrm{s}$. Two vehicles were used, V1 and V2, of masses $21.6 \mathrm{~kg}$ and $25.8 \mathrm{~kg}$ respectively. V1 and V2 had the same bounce frequencies of $2.93 \mathrm{~Hz}$ while pitch frequencies were $3.9 \mathrm{~Hz}$ and $3.7 \mathrm{~Hz}$ respectively.
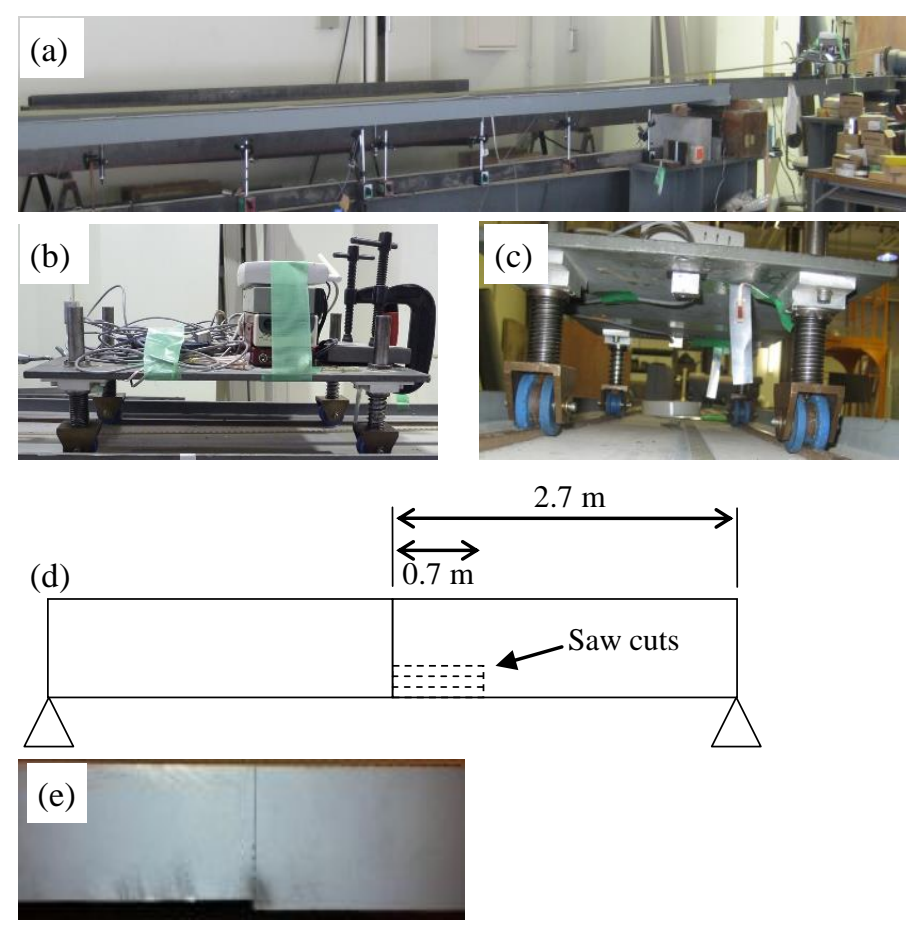

Figure 2. Experiment setup (a) Beam (b) Vehicle elevation (c) Vehicle end view (d) Beam elevation showing saw cut location (e) Saw cut

The scaled bridge model was a simply supported steel beam of span $5.4 \mathrm{~m}$ which incorporated a scaled road surface profile. It was fitted with accel- 
erometers at mid-span and quarter points to monitor its response during vehicle crossings, however this response is not analysed here. It had frequency $f_{b, \text { exp }}$ $=2.6 \mathrm{~Hz}$, mass per unit length of $52 \mathrm{~kg} / \mathrm{m}$ and stiffness of $120,700 \mathrm{~N} \mathrm{~m}^{2}$.

For the experiment, damage was applied via 0.7 $\mathrm{m}$ long rectangular saw-cuts in the beam's flanges between midspan and 3/8ths of the span. Four scenarios were investigated: Intact, D1, D2 and D3 corresponding to no damage, $5 \mathrm{~mm}, 10 \mathrm{~mm}$ and $15 \mathrm{~mm}$ cuts respectively (Figure 2(d),(e)). A sampling frequency of $100 \mathrm{~Hz}$ was used in the experiment.

\subsection{Continuous wavelet transform and damage indicator}

The real valued symmetrical Morlet wavelet basis is adopted for the CWT (Mallat 2008) in this study, as used by McGetrick \& Kim (2013), McGetrick \& Kim (2014) and described by Equation 1.

$\psi(t)=e^{-\frac{t^{2}}{2}} \cos (5 t)$

For the CWT, time localisation is an important criterion in order to detect damage location and the Morlet wavelet provides an appropriate balance between time and frequency resolution for this purpose. Therefore the Morlet CWT of vehicle accelerations is adopted as a damage sensitive feature.

The CWTs of the differences between healthy and damaged accelerations obtained in theoretical simulations and the experiment are analysed in both time and frequency domains simultaneously for the purpose of damage detection. All source acceleration signals are normalised using their standard deviations before calculating the differences and applying the CWT. Peaks occurring in the wavelet coefficients of the acceleration difference indicate the existence and location of damage. Damage indicators (DIs) based on the maximum magnitude of these peaks at particular frequencies are established. They focus on frequencies related to the vehicle - as they are the most dominant in the VBI - and also, those related to the bridge for comparative purposes. It should be noted that these DI's are calculated relative to the healthy bridge scenario.

\subsection{SPR technique overview}

For this investigation, repeated passes of the vehicle over the bridge are carried out in both simulations and the experiment. McGetrick \& Kim (2013) highlight the importance of the road profile roughness but assume the vehicle will always travel over the same road profile. Here, the possibility of accounting for road profile variability in the approach is investi- gated, utilizing recorded accelerations from repeated vehicle passes and a statistical pattern recognition approach is investigated. Instead of using a simple subtraction and relative DIs to measure the difference between damage sensitive features for healthy and damaged scenarios i.e. the damage extent, the Mahalanobis distance (MD) is used for repeated bridge crossings in a 2 dimensional analysis. The MD is used in multivariate analysis to measure the distance or separation between datasets (Bishop 2006, Nair and Kiremidjian 2007) and here, is defined by Equation 2, where $x$ and $\mu$ define means of datasets and subscripts $h$ and $d$ indicate healthy and damaged data respectively. $\Sigma_{h}$ is the covariance matrix for the healthy data. MD is incorporated in this study as a damage extent measure to investigate its effectiveness and suitability for inclusion in the original approach.

$$
M D=\sqrt{\left(x_{h}-\mu_{d}\right)^{\mathrm{T}} \Sigma_{h}^{-1}\left(x_{h}+\mu_{d}\right)}
$$

In this paper, absolute DIs for axle 1 and axle 2 of the vehicle are taken as variables for the MD. These DIs are based on the maximum magnitude of the CWT of accelerations for each damage scenario tested, including the healthy scenario, thus they are not relative values. It follows that these absolute DIs are intended to be used for global damage detection and cannot identify damage location. The number of observations, $n$, in the data used for MD, depends on the number of bridge crossings. In simulations $n=$ 51 which is the number of road profiles tested. In the experiment, $n=5$ which is the number of repeated tests for each vehicle, speed and damage scenario.

\section{RESULTS AND DISCUSSION}

\subsection{Theoretical simulation results}

In simulations, the aim is study the effectiveness of the existing wavelet-based drive-by system in identifying the existence and location of damage in a bridge for a range of parameters and to examine the effectiveness of combining it with an alternative SPR technique, briefly described in Section 2.4. A bridge span length of $15 \mathrm{~m}$, vehicle speeds of $2 \mathrm{~m} / \mathrm{s}$, $5 \mathrm{~m} / \mathrm{s}, 10 \mathrm{~m} / \mathrm{s}$ and $20 \mathrm{~m} / \mathrm{s}$, a perfectly smooth profile and a rough road profile carpet of 51 correlated road profiles are tested. The damage level and location are also varied; stiffness reductions from $1 \%$ up to $20 \%$ are applied to an individual beam element at $3 L / 8$ or $L / 2$. In the following figures of CWT coefficients, solid parallel vertical lines indicate the entry/exit time of the axle on the damaged beam element while the crossing dashed lines mark the time and frequency of the maximum wavelet coefficient. Results are presented in this section for measure- 
ments above axle 2 only, $\ddot{y}_{s, 2}$, unless otherwise stated; similar results were found for those above axle 1 .

\subsubsection{Effect of road roughness and vehicle speed}

Figures 3(a) and 3(b) illustrate the CWT coefficients of vehicle accelerations for a perfectly smooth road profile and a rough road profile respectively. Results shown correspond to a rough road profile which has been selected from a randomly generated profile carpet of 51 correlated 'very good' class A profiles (ISO 1995) that are tested in simulations. It can be seen that that for both smooth and rough road profiles, $1 \%$ damage in an element at midspan is detected globally but can also be located in the wavelet coefficients at peaks which correspond to the vehicle frequency of $1.4 \mathrm{~Hz}$. As noted by McGetrick \& Kim (2013), this indicates that it should be beneficial to formulate damage indicators based on this frequency response. However, it should also be noted for the rough road profile that the magnitudes of the coefficients are much lower and the damage location is not detected as accurately.

Figure 3(c) highlights that as vehicle speed increases to $5 \mathrm{~m} / \mathrm{s}$, the time and frequency resolutions decrease considerably due to the shorter VBI time. However, the maximum peak magnitude has not changed considerably and damage can still be detected. As speed is increased further, it is found that location accuracy is less reliable although global changes due to the damage are detected.

\subsubsection{Effect of damage level and location}

Figure 4(a) illustrates the CWT coefficients for vehicle accelerations considering $1 \%$ damage at $3 L / 8$ for a rough road profile while Figure 4(b) shows the corresponding values for a higher damage level of $5 \%$; the peak magnitudes at the damage location increase proportionally with respect to the damage level. The location of the peaks does not vary with increased damage level. Also, comparing Figure 4(a) to Figure 3(b) for a rough road profile, it can be seen that damage is detected at a peak closer to the true damage location for $3 L / 8$, although the magnitude at this peak has decreased by about half compared to that for $L / 2$.

\subsubsection{Damage indicators}

Following the original approach by McGetrick \& Kim (2013), a damage indicator (DI1) based on the maximum coefficient on the frequency range corresponding to the vehicle, $\left[f_{v, 2} / 2,\left(f_{v, 2}+f_{b, 1}\right) / 2\right]$, is calculated and plotted in Figure 5(a) for all speeds and 51 rough road profiles in the carpet tested in simulations. To allow comparison between the DIs for all damage levels, they are standardised for each speed using their means and standard deviations. In addition to DI1 focusing on vehicle frequencies, another damage indicator, DI2, is formulated similarly based on the maximum coefficient on a frequency range re- lated to the bridge's $1^{\text {st }}$ natural frequency: $\left[\left(f_{v, 2}\right.\right.$ $\left.\left.+f_{b, 1}\right) / 2,1.27 f_{b, 1}\right]$. The corresponding results are plotted on Figure 5(b); DI1 and DI2 both allow damage levels to be distinguished from one another irrespective of the vehicle speed or road profile. For all damage levels at $L / 2$, the average errors in identified location for all 51 profiles tested, as a percentage of bridge span length, using DI1 are $10.1 \%, 12.5 \%$, $11.7 \%$ and $6.9 \%$ corresponding to speeds of $2 \mathrm{~m} / \mathrm{s}, 5$ $\mathrm{m} / \mathrm{s}, 10 \mathrm{~m} / \mathrm{s}$ and $20 \mathrm{~m} / \mathrm{s}$ respectively. The corresponding average errors for DI2 are $9.9 \%, 8.4 \%$, $17.6 \%$ and $11.3 \%$ respectively.
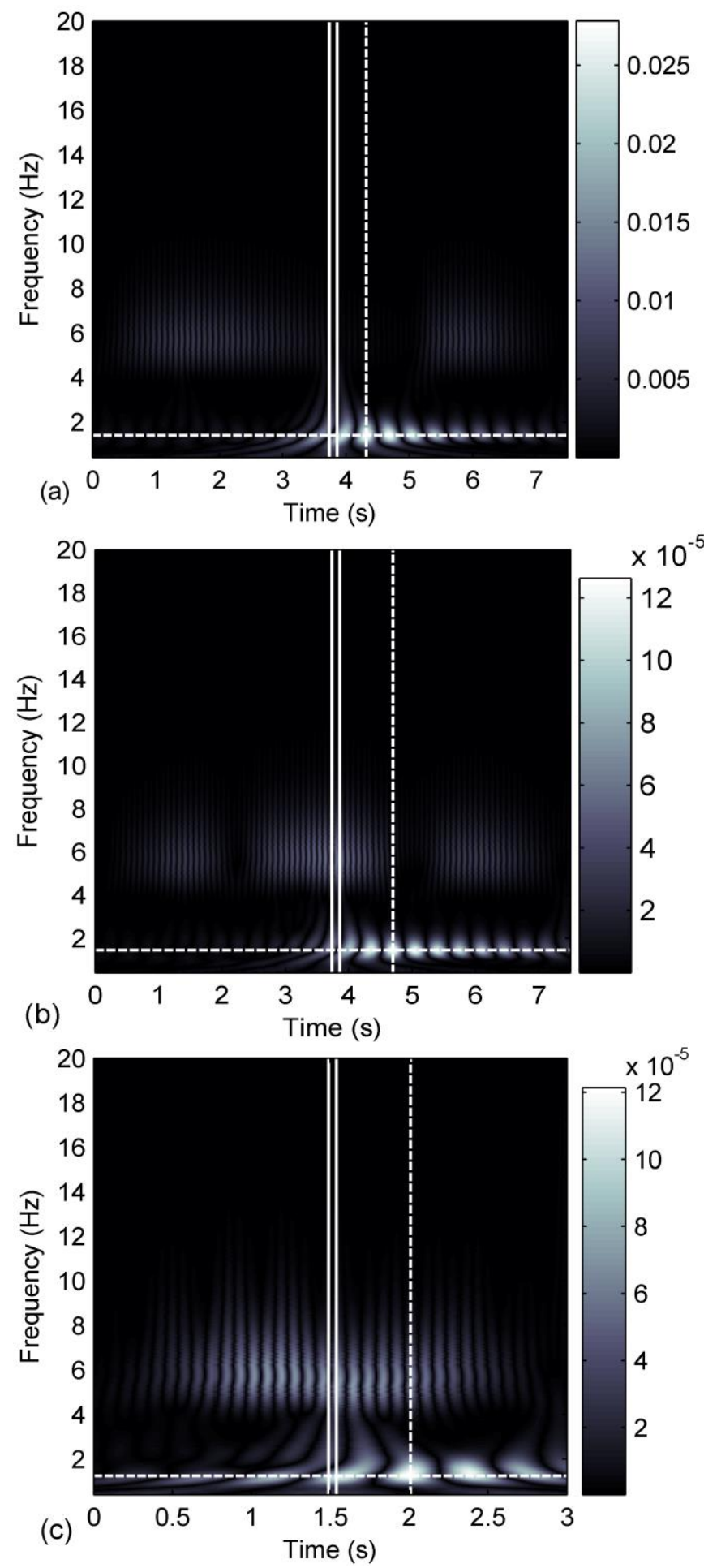

Figure 3 Wavelet coefficients of acceleration difference above axle 2 for $15 \mathrm{~m}$ span (a) perfectly smooth profile, $2 \mathrm{~m} / \mathrm{s}$ (b) ISO class A profile, $2 \mathrm{~m} / \mathrm{s}$ (c) class A profile, $5 \mathrm{~m} / \mathrm{s} ; 1 \%$ damage at $L / 2$. 
For damage at $3 L / 8$, the corresponding damage indicators are plotted in Figure 6, showing similar results to those in Figure 5. Average errors in identified damage location for DI1 and damage at $3 L / 8$ are $10 \%, 29 \%, 15.3 \%$ and $11.9 \%$ respectively. For DI2, the corresponding errors are $19 \%, 20.3 \%, 28.8 \%$ and $25.9 \%$ respectively. In general, the damage location for damage at $3 L / 8$ is identified less accurately than that at $L / 2$. This occurs as the peaks relating to this damage are of smaller magnitude in the CWT coefficients, as shown in Section 3.1.2, and hence are relatively more sensitive to changes in the road profile. In addition, in Figures 3 and 4, the largest bridge frequency related vehicle response does not appear to be as sensitive to the damage location compared to the vehicle frequency related response. This suggests that although DI2 may be useful for detecting and quantifying damage, DI1 is more suitable for identifying the damage location.
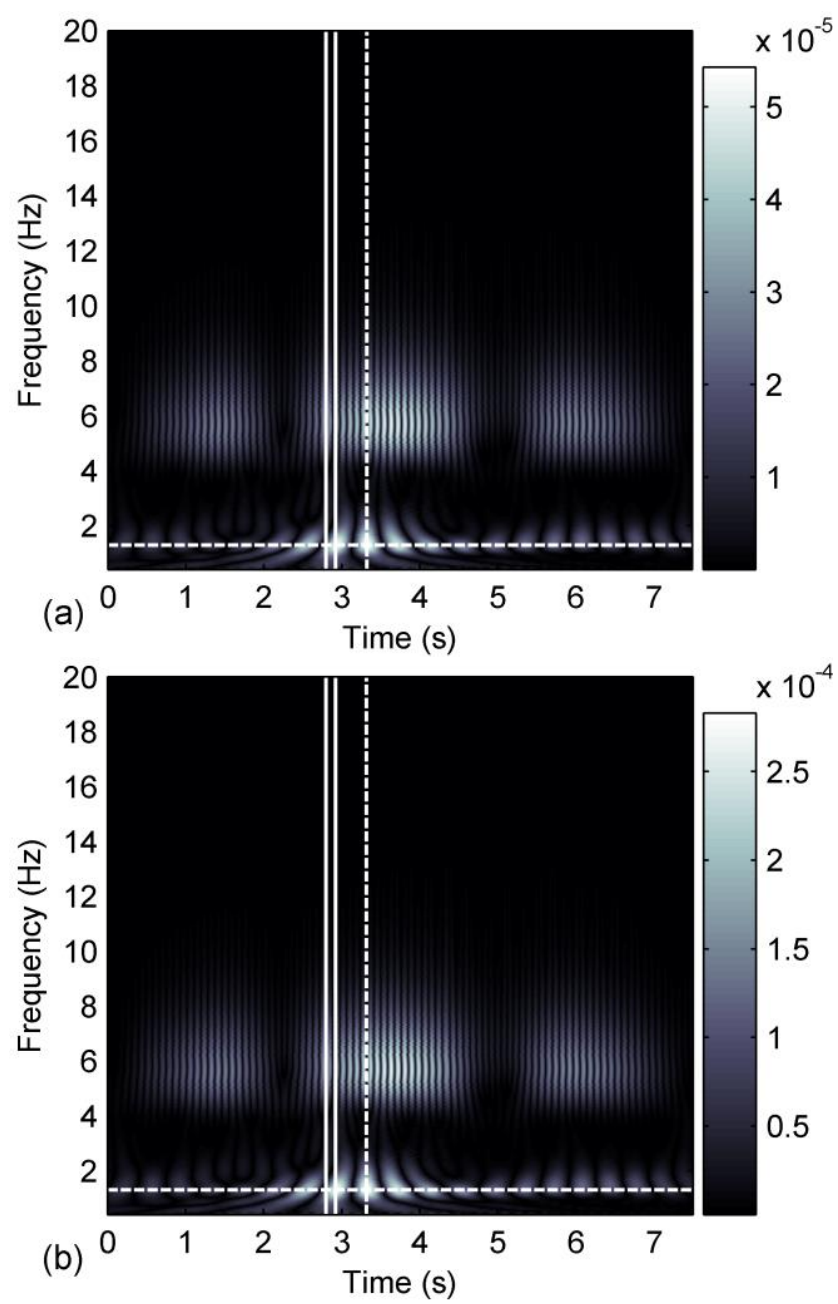

Figure 4 Wavelet coefficients of acceleration difference above axle 2 for $15 \mathrm{~m}$ span (a) $1 \%$ damage at $3 L / 8$ (b) $5 \%$ damage at $3 L / 8$; ISO class A profile, speed is $2 \mathrm{~m} / \mathrm{s}$.
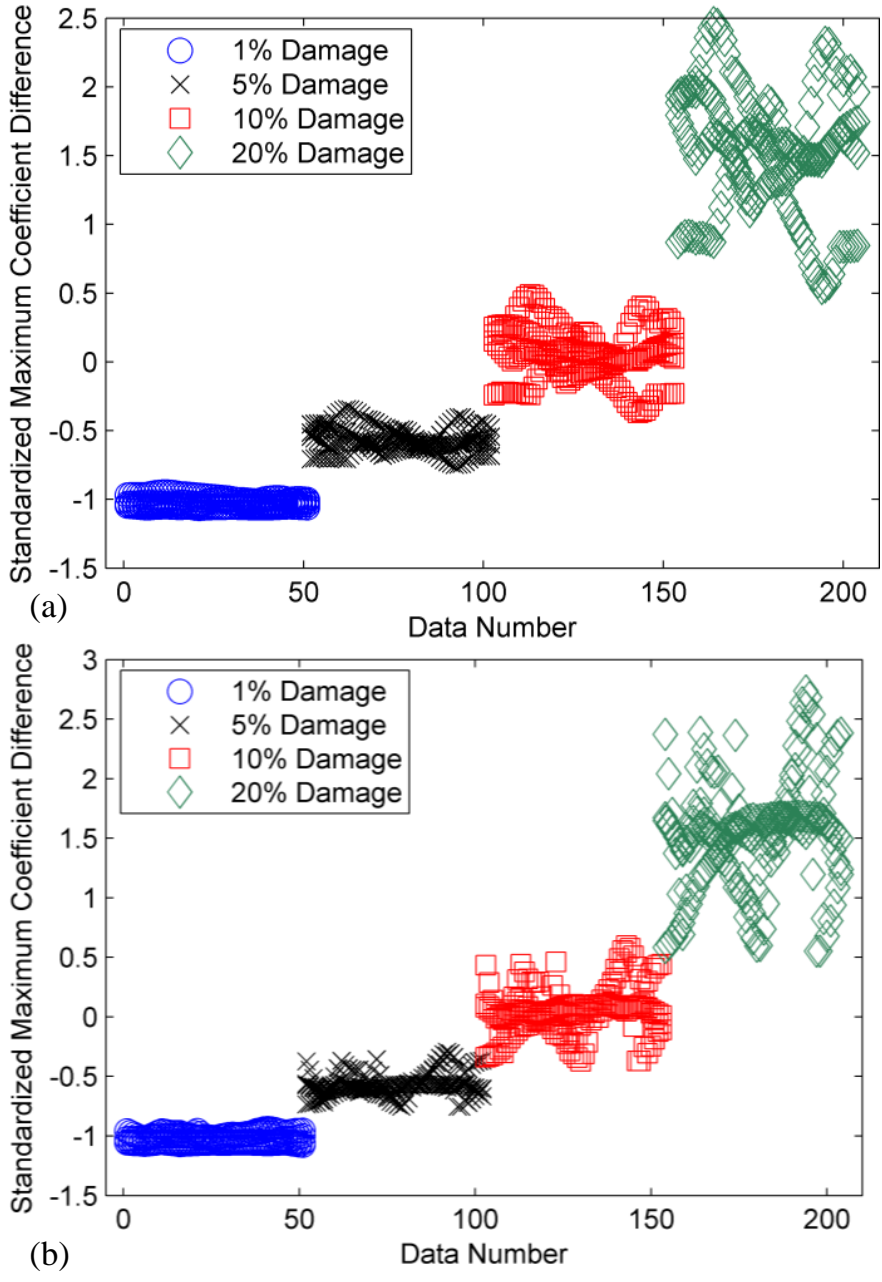

Figure 5 Damage indicators for damage at $L / 2$ (a) DI1 (b) DI2
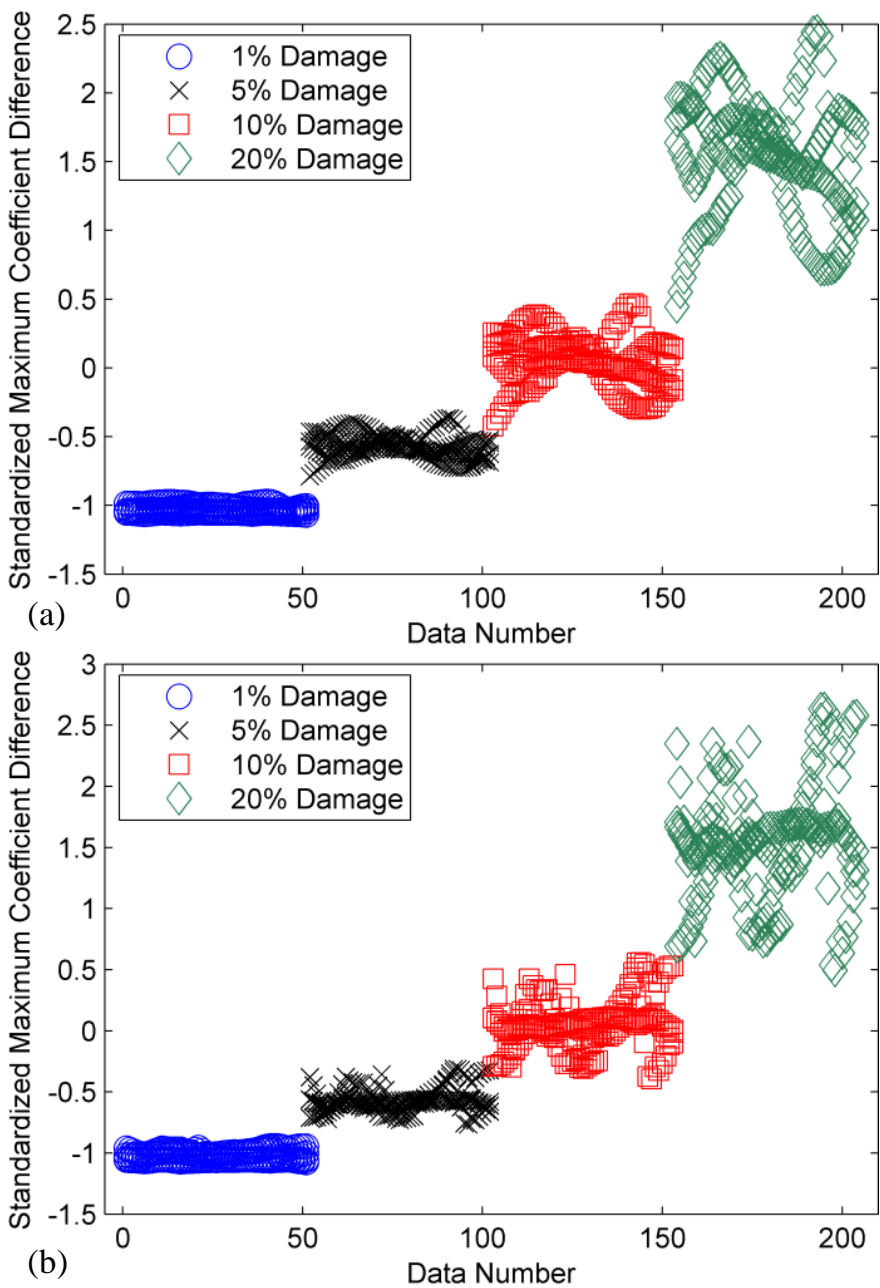

Figure 6 Damage indicators for damage at $3 L / 8$ (a) DI1 (b) DI2 


\subsubsection{Results of SPR technique using MD}

For the SPR technique utilising MD presented in Section 2.4, the DIs (or variables) used for axles 1 and 2 in each damage scenario are the maximum CWT coefficients of axle accelerations within the frequency range $\left[\left(f_{v, i}+f_{b, 1}\right) / 2,1.27 f_{b, 1}\right]$, where $i=1,2$. The values of the MDs calculated based on these DIs for all damage levels and road profiles for a speed of $2 \mathrm{~m} / \mathrm{s}$ are plotted in Figure 7 for damage located at $L / 2$. Similar results can be observed for higher speeds and damage located at $3 L / 8$. To provide a reference for the MDs, the MDs of the all points in the healthy dataset relative to its own mean are also plotted.

All of the data points appear superimposed upon each other for each damage level while the differences between MDs caused by damage level are insignificant compared to the changes due to the road profile. These results indicate that even a slight variation in road profile can mask the effects of damage on the selected indicator. It also indicates that although the MD can be incorporated here for repeated passes, the selected absolute DIs extracted from vehicle measurements at axle 1 and axle 2 are not sensitive enough to the bridge damage at midspan and are a poor choice. This highlights a significant challenge to overcome for implementation of this approach in practice. Although relative DIs based on the difference between the CWT coefficients shown in Figures 5 and 6 can distinguish between damage scenarios for the vehicle travelling on the same road profile, this damage sensitivity may also be too small in practice where, unless highly controlled, variations in road position can occur.

\subsection{Experiment results}

In this section, results are presented for accelerations recorded at axle 2 of the experimental vehicle model V1 only; despite the increase in mass, similar results were obtained for vehicle V3 and axle 1. Figure 8 shows an example of the CWT coefficients obtained for axle 2 of vehicle V1 and speed S2 $(1.16 \mathrm{~m} / \mathrm{s})$; the maximum peak is marked by the dashed white lines in this figure, occurring at peaks corresponding to the vehicle pitch frequency of $3.9 \mathrm{~Hz}$.

\subsubsection{Experimental damage indicators}

To account for the vehicle frequencies $(2.93 \mathrm{~Hz}, 3.7$ $\mathrm{Hz}$ and $3.9 \mathrm{~Hz}$ ) being higher than that of the bridge $(2.6 \mathrm{~Hz})$, the frequency range used for the damage indicators is changed. DI1 becomes DI1 $1_{\exp }$ and its frequency range is adjusted to $\left[0.73 f_{b, \exp }, \quad\left(f_{v, 2}\right.\right.$ $\left.\left.+f_{b, \exp }\right) / 2\right]$. DI 2 becomes DI $2_{\text {exp }}$ and its updated frequency range is $\left[\left(f_{v, 2}+f_{b, \text { exp }}\right) / 2,1.27 f_{v, 2}\right]$. The experimental damage indicators are plotted in Figure 9 for vehicle 1, all speeds and repeated tests. It can be clearly seen that compared to simulations, it is much more difficult to distinguish between damage scenar- ios in the experiment, although focusing on the bridge frequency (Figure 9(b)) appears to provide a greater chance of doing so.

For DI $1_{\text {exp }}$, average percentage errors in identified damage location (relative to the edge of the saw cut segment) for all damage scenarios were $28.5 \%$, $20.3 \%$ and $21.7 \%$ for speeds of $0.93 \mathrm{~m} / \mathrm{s}, 1.16 \mathrm{~m} / \mathrm{s}$ and $1.63 \mathrm{~m} / \mathrm{s}$ respectively. For DI $2_{\exp }$, the corresponding percentage errors were $22.7 \%, 22.3 \%$ and $21 \%$ respectively and are comparable in terms of magnitude to the errors obtained for DI2 with damage at $3 L / 8$ in simulations.

Neither DI in the experiment showed a strong relationship with speed, indicating that speed may not be as critical a factor in identifying damage location as suggested in Section 3.1.1 and in previous studies. Although these errors are around 20\%, in practice this would allow inspectors to focus on a particular section of a bridge span for damage. Nonetheless, obtaining more accurate and precise results is a challenge that needs to be overcome in order to clearly distinguish between damage levels.

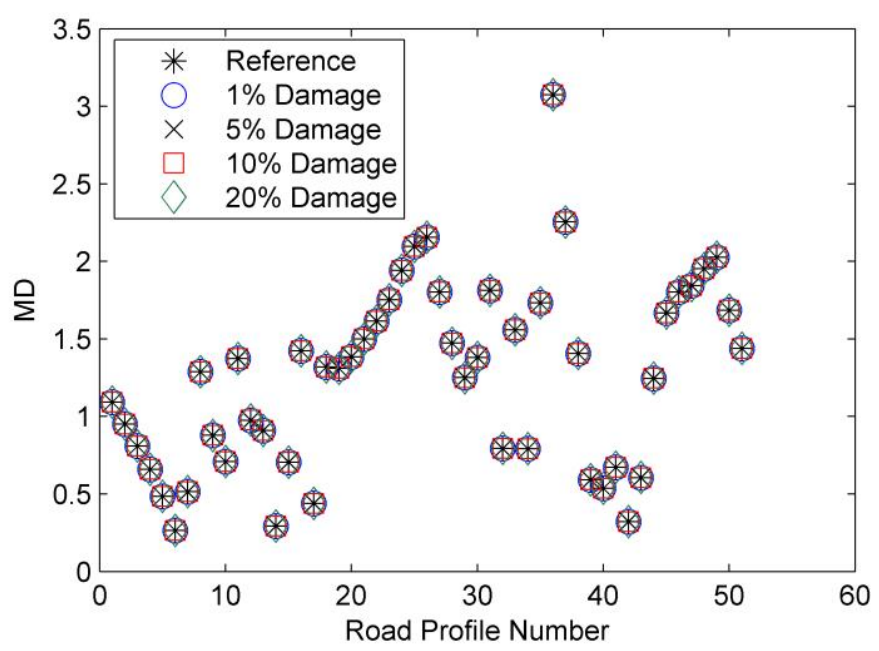

Figure 7 Mahalanobis distances calculated for a vehicle speed of $2 \mathrm{~m} / \mathrm{s}$; damage located at $L / 2$.

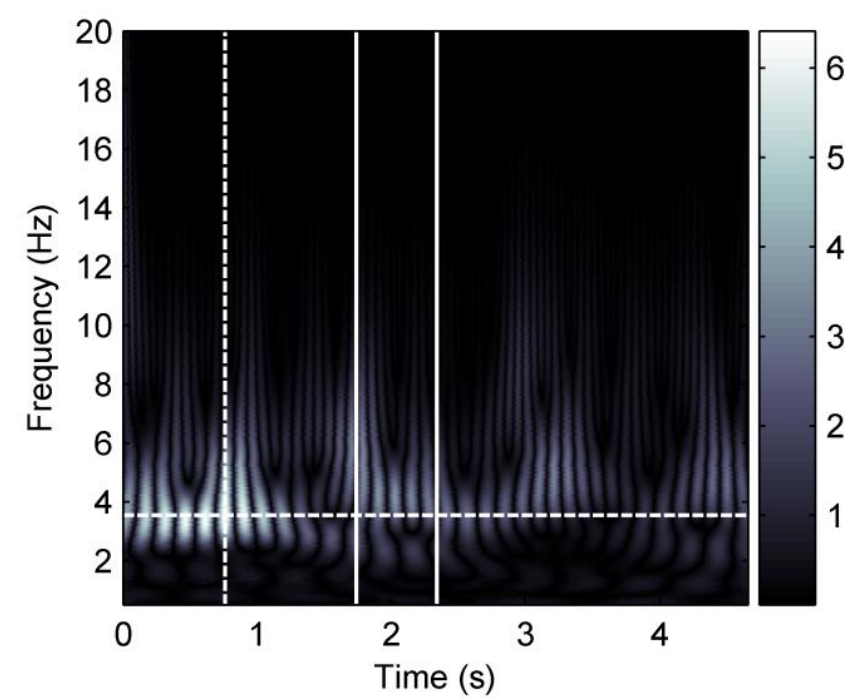

Figure 8 Wavelet coefficients of acceleration difference above axle 2 on vehicle $\mathrm{V} 1$ for speed $\mathrm{S} 2=1.16 \mathrm{~m} / \mathrm{s}$. 

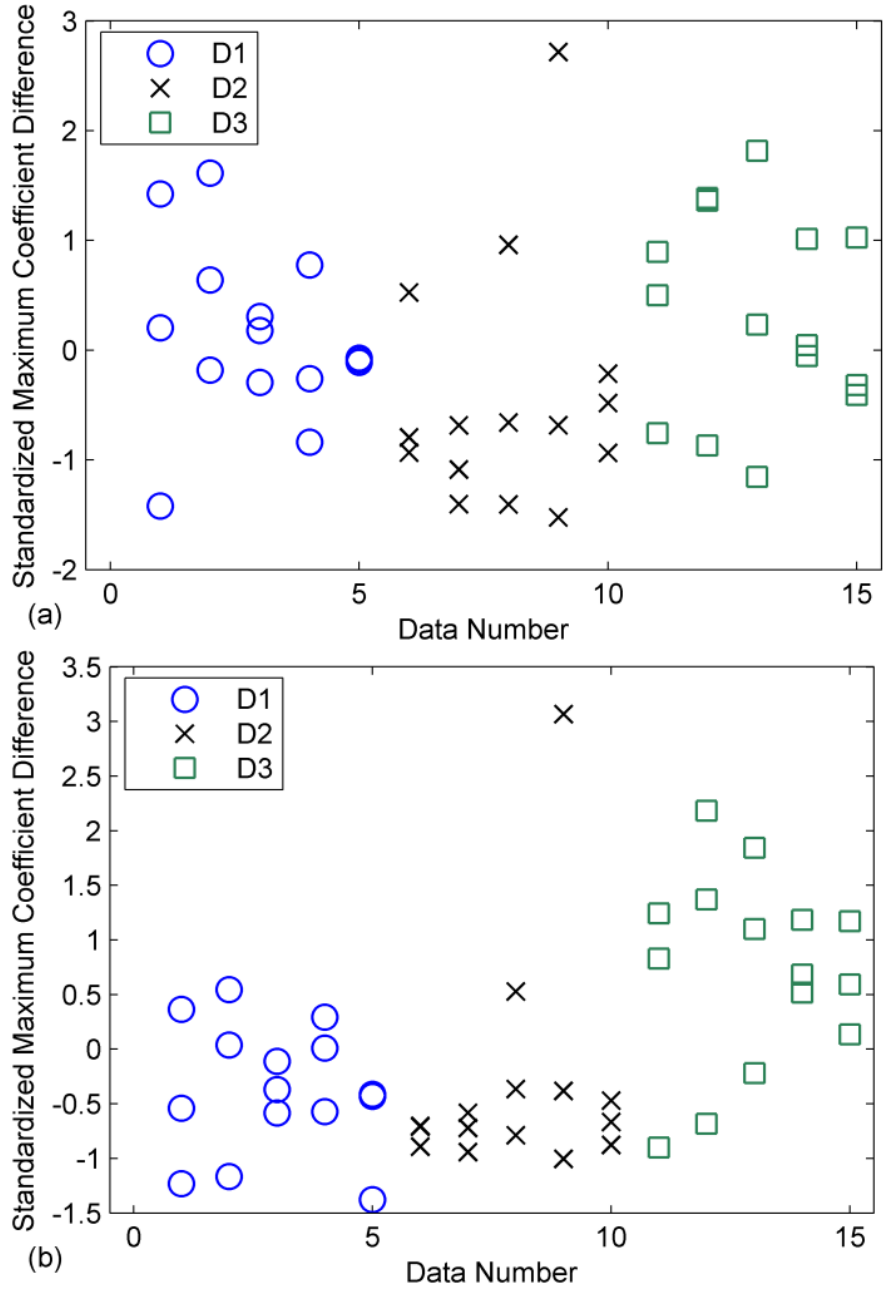

Figure 9 Damage indicators for vehicle V1 (a) DI $1_{\exp }$ (b) DI $2_{\text {exp. }}$

\subsubsection{Application of SPR technique and MD}

The application of the SPR technique using MD in simulations highlighted that variation in road profile between bridge crossings is a challenge that needs to be overcome. In the laboratory experiment, the same road profile was used for every bridge crossing thus no variation in road profile occurred. Hence, results are presented in this section for the same SPR technique applied to the experimental data. The DIs used for MD calculation for axle 1 and axle 2 are obtained in a similar fashion to those in simulations; in the experiment the frequency range is $\left[0.73 f_{b, \exp },\left(f_{v, i}\right.\right.$ $\left.+f_{b, \exp }\right) / 2$ ] for axle $i=1,2$.

The values of the MDs for all 4 damage levels and 5 tests for speed $\mathrm{S} 2=1.16 \mathrm{~m} / \mathrm{s}$ are plotted in Figure 10 for vehicle V1. Once again, to provide a reference for the MDs, the MDs of the all points in the healthy or 'Intact' dataset relative to its own mean are also plotted. It can be seen that in general, the damaged scenarios have higher MDs than the Intact case, thus the damage can be detected. In addition, the MD tends to increase with increasing damage level i.e. greater saw cut depth in the beam. Taking the mean of the 5 tests would allow damage and its severity to be identified. Similar results are observed for other speeds and vehicle V3.

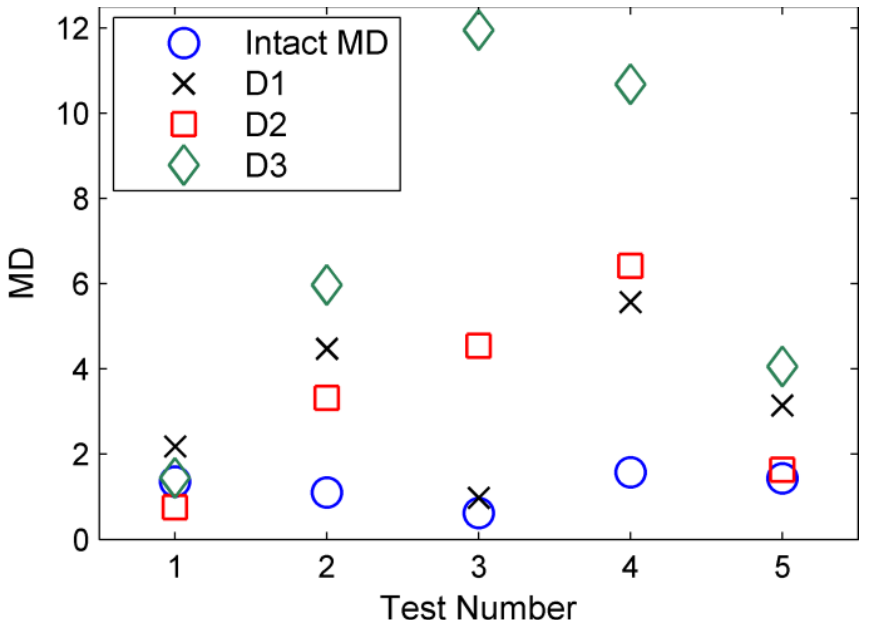

Figure 10 Mahalanobis distances calculated for vehicle V1 and speed $\mathrm{S} 2=1.16 \mathrm{~m} / \mathrm{s}$.

Table 1 summarises the mean MDs for all vehicles, damage scenarios and vehicle speeds. Vehicle V1 appears to be more sensitive to increases in damage level and this is most likely due to its vibration frequency characteristics. Overall, considering results for simulations shown in Figure 7, these experimental results suggest that in practice, if the vehicle's road position is controlled, this SPR technique could detect bridge damage and distinguish between different levels of damage, irrespective of vehicle speed.

Table 1. Mean Mahalanobis distances for experimental damage scenarios

\begin{tabular}{lllll}
\hline Vehicle & \multicolumn{4}{l}{ Damage Scenario } \\
\cline { 2 - 5 } & Intact & D1 & D2 & D3 \\
\hline V1 & 1.1617 & 2.7178 & 3.2141 & 4.4359 \\
V2 & 1.2035 & 2.4494 & 3.0157 & 3.1015 \\
\hline
\end{tabular}

\section{CONCLUSIONS}

This paper presents both numerical and experimental investigations of a wavelet based drive-by system for bridge monitoring, incorporating a vehicle fitted with accelerometers. In simulations, results for the identification of the existence and location of damage align with those of previous studies; damage is detected less accurately for higher speed and rough road profiles, while differences in the magnitudes of wavelet coefficients of vehicle accelerations can indicate the level of damage. Formulating damage indicators based on these magnitudes allows different damage levels to be distinguished provided the same profile is crossed on the bridge each time. However, studying damage indicators using absolute coefficient magnitudes and incorporating the Mahalanobis distance as a damage extent measure, it is shown that variation in road profile can have a greater effect on global VBI responses than the damage itself.

In a laboratory experiment damage can be detected but it is found to be much more difficult to distin- 
guish between damage scenarios using the damage indicators. Experimental results also highlight that the SPR technique incorporating MD can be effective for detecting damage and providing a measure of its severity if the vehicle's position on the bridge is controlled.

This approach has potential to incorporate a range of statistical pattern recognition techniques. In practice, this drive-by system could be used target periodic monitoring of short to medium span highway bridges, carried out at highway vehicle speeds. This could support a long term monitoring database for a road network. Successful development and implementation of this system would allow more effective resource allocation by highlighting bridges which are in need of more detailed inspection and maintenance. Further to this, the drive-by system could be redeployed for such bridges at slower speed, incorporating a pattern-adapted wavelet for increased damage location detection accuracy. Further work focused on field trials and optimal selection of damage sensitive features is required to address challenges associated with this real-world application of the system.

\section{ACKNOWLEDGEMENTS}

The authors wish to express their gratitude for the financial support received from the Japanese Society for the Promotion of Science for the Grant-in-Aid for Scientific Research (B) under project no. 24360178.

\section{REFERENCES}

Bishop, C.M. 2006. Pattern Recognition and Machine Learning. New York: Springer.

Boller, C., Chang, F.K. \& Fujino, Y. 2009. Encyclopedia of Structural Health Monitoring. West Sussex, UK: John Wiley \& Sons Ltd.

Bu, J.Q., Law, S.S. \& Zhu, X.Q. 2006. Innovative bridge condition assessment from dynamic response of a passing vehicle. Journal of Engineering Mechanics, ASCE 132(12): 1372-1379.

Carden, E.P. \& Fanning, P. 2004. Vibration based condition monitoring: A review. Structural Health Monitoring, 3(4): 355-377.

González, A., Covián, E. \& Madera, J. 2008. Determination of Bridge Natural Frequencies Using a Moving Vehicle Instrumented with Accelerometers and GPS. Proceedings of the Ninth International Conference on Computational Structures Technology, Athens, Greece, Paper 281.

González, A., OBrien, E.J. \& McGetrick, P.J. 2012. Identification of damping in a bridge using a moving instrumented vehicle. Journal of Sound and Vibration 331(18): 41154131.

ISO 8608. 1995. Mechanical Vibration-road Surface Profilesreporting of Measured Data. International Standards Organisation.

Keenahan, J., McGetrick, P.J., OBrien, E.J. \& González, A. 2012. Using Instrumented Vehicles to Detect Damage in
Bridges, Proceedings of the 15th International Conference on Experimental Mechanics, Porto, Portugal, Paper 2934.

Khorram, A., Bakhtiari-Nejad, F. \& Rezaeian, M. 2012. Comparison studies between two wavelet based crack detection methods of a beam subjected to a moving load. International Journal of Engineering Science 51: 204-215.

Kim, CW, Isemoto, R, Toshinami, T, Kawatani, M, McGetrick, PJ, \& OBrien, E.J. 2011. Experimental investigation of drive-by bridge inspection. 5th International Conference on Structural Health Monitoring of Intelligent Infrastructure (SHMII-5), Dec. 11-15, Cancun, Mexico.

Lin, C.W. \& Yang, Y.B. 2005. Use of a passing vehicle to scan the fundamental bridge frequencies. An experimental verification. Engineering Structures 27: 1865-1878.

Mallat, S. 2008. A Wavelet Tour of Signal Processing, Third Edition: The Sparse Way. USA: Academic Press.

McGetrick, P.J., González, A. \& OBrien, EJ. 2009. Theoretical investigation of the use of a moving vehicle to identify bridge dynamic parameters. Insight 51(8): 433-438.

McGetrick, P.J., Kim, C.W., González, A. \& OBrien, E.J. 2013. Dynamic Axle Force and Road Profile Identification using a Moving Vehicle. International Journal of Architecture, Engineering and Construction 2(1): 1-16.

McGetrick, P.J. \& Kim, C.W. 2013. A parametric study of a drive by bridge inspection system based on the Morlet wavelet. Key Engineering Materials 569-570: 262-269.

McGetrick, P.J. \& Kim, C.W. 2014. A wavelet based drive-by bridge inspection system. Proceedings of the Seventh International Conference on Bridge Maintenance, Safety and Management, IABMAS2014, Shanghai, China, Paper 0159.

Mesa, H. 2005. Adapted Wavelets for Pattern Detection. In Sanfeliu, A. \& Cortés, M. (eds), Progress in Pattern Recognition, Image Analysis and Applications. Lecture Notes in Computer Science 3773: 933-944. Heidelberg: Springer Berlin.

Miyamoto, A. \& Yabe, A. 2012. Development of practical health monitoring system for short- and medium-span bridges based on vibration responses of city bus. Journal of Civil Structural Health Monitoring 2: 47-63.

Nair, K.K. \& Kiremidjian, A.S. 2007. Time Series Based Structural Damage Detection Algorithm Using Gaussian Mixtures Modeling. Journal of Dynamic Systems, Measurement, and Control, ASME, 129: 285-293.

Nair, K.K. \& Kiremidjian, A.S. 2009. Derivation of a Damage Sensitive Feature Using the Haar Wavelet Transform. Journal of Applied Mechanics, ASME 76(6): 1-9.

Nguyen, K.V. \& Tran, H.T. 2010. Multi-cracks detection of a beam-like structure based on the on-vehicle vibration signal and wavelet analysis. Journal of Sound and Vibration 329(21): 4455-4465.

Reda Taha, M.M., Noureldin, A., Lucero, J.L. \& Baca, T.J. 2006. Wavelet transform for structural health monitoring: a compendium of uses and features. Structural Health Monitoring 5(3): 267-295.

Tedesco, J.W., McDougal, W.G. \& Ross, C.A. 1999. Structural Dynamics, Theory and Applications. Boston, United States: Addison-Wesley.

Yang, Y.B., Lin, C.W. \& Yau, J.D. 2004. Extracting bridge frequencies from the dynamic response of a passing vehicle. Journal of Sound and Vibration 272: 471-493.

Yang, Y.B. \& Chang, K.C. 2009. Extracting the bridge frequencies indirectly from a passing vehicle: Parametric study. Engineering Structures 31(10): 2448-2459. 